\title{
Comparação entre a Bomba de Infusão de Insulina Subcutânea e o Esquema de Múltiplas Doses de Insulina em Adolescentes com Diabetes Melito do Tipo 1 da Rede Pública de Saúde na Abordagem da Hipoglicemia Grave
}

\section{RESUMO}

Objetivo: Avaliação comparativa da freqüência de hipoglicemia severa após mudança da terapia com múltiplas doses de insulina (MDI) para bomba de insulina subcutânea (BIISC). Pacientes e Métodos: Sete pacientes $\mathrm{DM} 1$, idade $\mathrm{M} i=14$ anos e tempo médio de diabetes de 8 anos, comparados de acordo com a incidência de hipoglicemia, a dose total de insulina (U/Kg/d), IMC ( $\left.\mathrm{Kg} / \mathrm{m}^{2}\right)$ e HbA1c (vn: 3,5-6,7\%) 1 ano antes e 1 ano após a transferência de terapêutica. Houve redução significativa dos episódios de hipoglicemia severa (1,3 episódio/paciente/ano para zero episódio/paciente/ano; $p=0,04)$, na dose total diária de insulina $(1,33 \pm$ $0,26 \mathrm{U} / \mathrm{Kg} / \mathrm{dia}$ para $0,87 \pm 0,17 \mathrm{U} / \mathrm{kg} / \mathrm{dia} ; \mathrm{p}=0,04)$ e na $\mathrm{HbA} 1 \mathrm{c}(8,7 \pm 0,7 \%$ para 7,8 $\pm 0,9 \% ; p=0,05$ com BIISC). Concluímos que a BIISC é eficaz e segura na redução de hipoglicemia severa em um subgrupo de pacientes DM1 com MDI. Entretanto, os resultados obtidos precisam ser reproduzidos em centros semelhantes e estudos de custo são necessários para que a sua viabilidade seja confirmada e aplicada nos sistemas públicos de saúde, que correspondem à maioria no nosso país. (Arq Bras Endocrinol Metab 2007;51/7:1169-1174)

Descritores: Diabetes mellitus tipo 1; Múltiplas doses de insulina; Bomba de infusão de insulina subcutânea; Hipoglicemia; Adolescentes

\section{ABSTRACT}

Comparison Between Continuous Subcutaneous Insulin Infusion and Multiple Daily Injection Regimens in Adolescent with Type 1 Diabetes from a Public Health Care System Approach to Severe Hypoglycemia.

Objective: We compared the incidence of severe hypoglycemia episodes with therapy with multiple doses of insulin (MDI) and after changing to pump (CSII). Patients and methods: 7 T1DM patients with 14 years median and median duration of diabetes of 8 years. We analyzed insulin requirement (U/kg/day), $\mathrm{BMI}\left(\mathrm{Kg} / \mathrm{m}^{2}\right), \mathrm{HbA} 1 \mathrm{c}$ (normal range: $3.5-6.7 \%$ ) one year before and one year after changing therapy. The severe hypoglycemia episodes decreased from 1.3 to 0 episodes/patient/year; $p=0.00$ ). The insulin requirement decreased from $1.33 \pm 0.26 \mathrm{U} / \mathrm{Kg} /$ day to $0.87 \pm 0.17$ $\mathrm{U} / \mathrm{kg} / \mathrm{day} ; \mathrm{p}=0.04$ and $\mathrm{HbA} 1 \mathrm{c}$ decreased from $8.7 \pm 0.7 \%$ to $7.8 \pm 0.9 \% ; \mathrm{p}=$ 0.05 . Conclusion: $\mathrm{CSI}$ is efficient in decreasing severe hypoglycemia in a subgroup of T1DM using MDI also in Public Health Care System (PHCS) conditions. However, these finding should be reproduced by other Diabetes Care centers and cost studies are necessary to confirm the viability and possibility of this therapy, when necessary, to T1DM patients, which correspond to the majority of these individuals in our country, seeing in the PHCS. (Arq Bras Endocrinol Metab 2007;51/7:1169-1174)

Keywords: Type 1 diabetes mellitus; Multiple doses of insulin; Insulin pump; Hypoglycaemia; Adolescents apresentação de caso

\author{
MôNICA A.L. GabBay \\ SÉRGIO A. DIB
}

Centro de Diabetes - Disciplina de Endocrinologia,

Departamento de Medicina, UNIFESP/EPM, São Paulo, SP.

Recebido em 22/03/07 Revisado em 17/04/07 Aceito em 25/07/07 
A HIPOGLICEMIA GRAVE (que necessita de ajuda externa e/ou aplicação de glucagon) afeta 21\% dos pacientes com diabetes melito do tipo 1 (DMl) anualmente, com uma incidência de 1 a 1,6 episódio/ por paciente/ por ano (1). Além disso, está associada a um alto custo econômico e social (falta escolar, falta dos pais no trabalho, transporte e visitas hospitalares fora da rotina) (2). A busca de valores de HbAlc próximos dos valores normais, o que comprovadamente reduz as complicações crônicas do diabetes melito (DCCT) através da aplicação de insulina nas vias atuais, provoca uma elevação na incidência dos episódios de hipoglicemia (3). A freqüência destes episódios também está relacionada à faixa etária baixa, ao nível sócio econômico, à história de hipoglicemias prévias ou assintomáticas, à ingestão de álcool, à neuropatia autonômica e à insuficiência renal (4). É conhecido que o padrão socioeconômico da criança com diabetes melito interfere na aderência ao tratamento, no seu controle glicêmico e na freqüência com que apresenta readmissão hospitalar (5).

Nos últimos anos, vários estudos têm demonstrado que a bomba de infusão de insulina subcutânea (BIISC) é uma boa alternativa terapêutica em pacientes na faixa etária pediátrica e que é bem aceita por crianças e adolescentes (6-9). Os avanços técnicos, tanto nas BIISC como nos cateteres, entre outros aspectos como a redução no seu tamanho e dispositivos de segurança, têm permitido ampliar o seu uso na faixa etária pediátrica.

Os episódios freqüentes de hipoglicemias graves ou assintomática estão entre as principais indicações da BIISC nessa faixa etária. Esta indicação é reforçada pela heterogeneidade dos resultados na redução da hipoglicemia grave com a utilização do esquema de múltiplas doses de insulina (MDI), com os análogos de insulina de ação curta e lenta (10-15). Esses estudos têm mostrado que a utilização desses análogos reduzem as hipoglicemias leves, moderadas e noturnas, mas não as graves. No entanto, ressaltam que um número maior de dados nesse sentido são necessários. Esses dados são importantes também pela reconhecida elevação do custo do tratamento quando passamos das insulinas NPH para os análogos de insulina até a BIISC (16).

\section{OBJETIVO}

Neste estudo, nós comparamos a administração de insulina por via subcutânea contínua através da BIISC com outros esquemas de múltiplas doses de insulina para redução da hipoglicemia grave em pacientes com DMl da rede pública de saúde durante o período de 2 anos.

\section{PACIENTES E MÉTODOS}

Foram analisados sete pacientes portadores de DMl (critério Sociedade Brasileira de Diabetes) acompanhados no Centro de Diabetes da UNIFESP. O critério de inclusão foi ter permanecido com episódios de hipoglicemia grave após a terapia de múltiplas doses de insulina (MDI) e a monitoração da glicemia capilar domiciliar. A mediana de idade foi de 14 anos (variando de 11 a 21 anos), sendo cinco do sexo masculino e com tempo médio de doença de 8 anos. Todos os pacientes, exceto um, eram púberes (Tanner III a V). Um paciente (15 anos de DM) já apresentava complicações microvasculares: nefropatia (proteinúria de $320 \mathrm{mcg} / \mathrm{min} \mathrm{e}$ hipertensão) e retinopatia incipiente.

A maioria dos pacientes durante a terapia de MDI utilizou a insulina glargina associada à insulina lispro ou aspart, às refeições, e 2 pacientes utilizaram insulina humana NPH no desjejum pela manhã, almoço e à noite ao deitar, associada à insulina humana regular nessas refeições e quando necessário, para ajuste da glicemia.

Todos os pacientes e familiares receberam treinamento durante 2 meses quanto à contagem de carboidrato e sobre o manuseio e cuidados no uso da bomba MiniMed ${ }^{\circledR}$ 508C (Minimed, Medtronic, Sylmar, CA). Todos os pacientes realizaram pelo menos quatro glicemias capilares/dia, sendo a média de seis avaliações/dia.

A implantação do tratamento com a BIISC em todos os pacientes foi ambulatorial no Centro de Diabetes da UNIFESP. Depois da instalação da BIISC, as consultas foram semanais, quinzenais, mensais e posteriormente trimestrais, à medida que os pacientes se tornavam familiarizados com o sistema. Os pacientes dispunham de um contato telefônico nas 24 horas com um dos médicos (MALG) do estudo.

Os pacientes iniciaram e foram mantidos com insulina lispro (Humalog ${ }^{\circledR}$ ). Em todos os casos a insulina foi bem tolerada.

Para calcular as doses iniciais de insulina foram utilizadas as necessidades de insulina diárias nos esquemas anteriores. Essas doses foram programadas 50\% como basal e $50 \%$ como bolus prandiais (17).

Os pacientes em MDI são orientados inicialmente a corrigir glicemias superiores a $150 \mathrm{mg} / \mathrm{dl}$ com $1 \mathrm{U}$ a cada 50 mg acima do alvo e usar $1 \mathrm{U}$ de lispro ou regular a cada 15 gramas de carboidrato, baseado nas glicemias 2 horas após refeição. Posteriormente, este fator de sensibilidade foi corrigido individualmente de acordo com as glicemias capilares obtidas até obtenção da glicemia-alvo.

Durante terapia com BSCII, os pacientes tinham como alvo glicemia de jejum entre 80 a $120 \mathrm{mg} / \mathrm{dl}$, e 2 a 3 horas pósprandial até $140 \mathrm{mg} / \mathrm{dl}$. Todos os pacientes iniciaram relação insulina/carboidrato de 1:15 g ajustada individualmente conforme resposta da automonitorização glicêmica.

A troca dos cateteres foi recomendada a cada 3 dias ou nas seguintes circunstâncias: glicemia capilar superior a $300 \mathrm{mg} / \mathrm{dl}$ e presença de corpos cetônicos, glicemia > 300 $\mathrm{mg} / \mathrm{dl}$ que não respondia à administração de insulina com a BIISC e na presença de infecção no local do cateter. 
Os dados analisados neste estudo foram o índice de massa corpórea (peso/altura ${ }^{2}-\mathrm{kg} / \mathrm{m}^{2}$ ), HbAlc (High Pression Liquid Cromatography; vn: 3,5 a 5,8\%), dados relativos à insulinização como dose total diária de insulina, taxa de infusão máxima de insulina, número de basais, fator de sensibilidade, fator de contagem de carboidrato $(\mathrm{CH})$ e número de episódios de hipoglicemia grave durante o período do estudo. Hipoglicemia grave foi definida como glicemia menor que $40 \mathrm{mg} / \mathrm{d}$, associada à inconsciência e/ou convulsão (expressa como episódio/ paciente/ ano).

Os dados analisados sobre a terapia de MDI foram retrospectivos, sendo a hemoglobina glicada a média das últimas três medidas, e os relativos à BIISC foram prospectivos na mesma população.

\section{Análise estatística}

Os resultados são fornecidos em média \pm desvio-padrão, analisados através do software EPI info para Windows. As diferenças com um $\mathrm{p}<0,05$ foram consideradas como estatisticamente significantes.

\section{RESULTADOS}

\section{Dose de insulina}

A dose diária de insulina apresentou uma queda significativa $(\mathrm{p}=0,04)$ quando os pacientes passaram do esquema de MDI $(1,33 \pm 0,26 \mathrm{U} / \mathrm{Kg} /$ dia $)$ para a BIISC $(0,87 \pm 0,17 \mathrm{U} / \mathrm{kg} /$ dia $)$. Durante o tratamento com a BIISC, esta dose era dividida em $40-60 \%$ como basal e o restante em bolus nas refeições. As relações insulina/hidrato de carbono e os fatores de correção utilizados na BIISC estão ilustradas na tabela 1 .

\section{IMC}

O índice de massa corpórea não mostrou alteração significativa durante o uso da BIISC $\left(20,5 \pm 1,5 \mathrm{Kg} / \mathrm{m}^{2}\right)$ em relação ao período com $\mathrm{MDI}\left(20,4 \pm 1,7 \mathrm{Kg} / \mathrm{m}^{2}\right)$.

Tabela 1. Dose de insulina (U/Kg/dia) utilizada no basal, a relação insulina/ $\mathrm{CH}^{*}$ e o fator de correção** durante o tratamento com a BIISC nos indivíduos com DM1 estudados.

\begin{tabular}{lccc}
\hline Pacientes & Basal (U/Kg/d) & CH $^{*}$ & FC** \\
A & 0,6 & $1 / 13$ & $1 / 30$ \\
B & 0,6 & $1 / 20$ & $1 / 50$ \\
C & 0,5 & $1 / 10$ & $1 / 30$ \\
D & 0,4 & $1 / 8$ e $1 / 10$ & $1 / 40$ \\
E & 0,4 & $1 / 6$ e $1 / 10$ & $1 / 40$ \\
F & 0,5 & $1 / 10$ & $1 / 40$ \\
G & 0,4 & $1 / 10$ e $1 / 15$ & $1 / 50$ \\
\hline
\end{tabular}

* 1 unidade de insulina para cada determinada quantidade de hidrato de carbono.

* 1 unidade de insulina para determinado valor da glicemia capilar superior a $120 \mathrm{mg} / \mathrm{dl}$.

\section{HbA1c}

Os valores de HbAlc dos pacientes com DMl mostraram uma queda significante $(\mathrm{p}=0,05)$, de 0,9 pontos percentuais, com a transferência do esquema de MDI $(8,7 \pm 0,7 \%)$ para a BIISC $(7,8 \pm 0,9 \%)$. Estas comparações estão apresentadas na figura 1 .

\section{Hipoglicemia grave}

A incidência de hipoglicemias graves reduziu significantemente (de 1,3 para 0 episódios/ paciente/ ano; $\mathrm{p}=0,00)$ quando os pacientes passaram do esquema de MDI para a BIISC (figura 2). Durante o período de 12 meses de acompanhamento dos pacientes com DMl e uso de BIISC, 35\% destes apresentaram episódios de hipoglicemia leve ou assintomática $(50-70$ $\mathrm{mg} / \mathrm{dl}$ ) mas nenhum quadro de hipoglicemia grave.

\section{DISCUSSÃO E CONCLUSÕES}

A BIISC é uma terapia factível em adolescentes assistidos nas condições de um serviço público de saúde e reduziu significativamente a incidência de hipoglicemia grave, as necessidades diárias de insulina, os valores de HbAlc e não provocou aumento no ganho de peso nesses pacientes.

Muitos estudos confirmam a diminuição da hipoglicemia grave com a infusão contínua de insulina subcutânea comparada à terapia insulínica por injeções, muitas vezes alcançando reduções até de 70\% (18-21). Vale ressaltar que os valores encontrados nestes estudos diferem muito, principalmente por nem sempre se definir corretamente hipoglicemia grave, isto é, a necessidade de ajuda de terceiros e/ou uso de

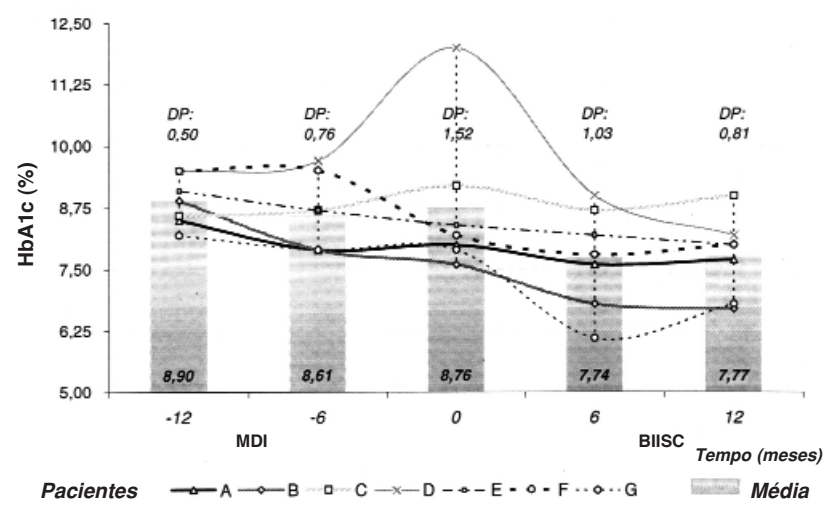

Figura 1. Valores individuais e média de $\mathrm{HbA} 1 \mathrm{c}(\%)$ durante o tratamento com MDI ( 1 ano antes $=-1 ; 6$ meses antes $=-0,5$ ) e com BIISC (após 6 meses = 0,5 e 1 ano) dos pacientes com DM1 estudados. 


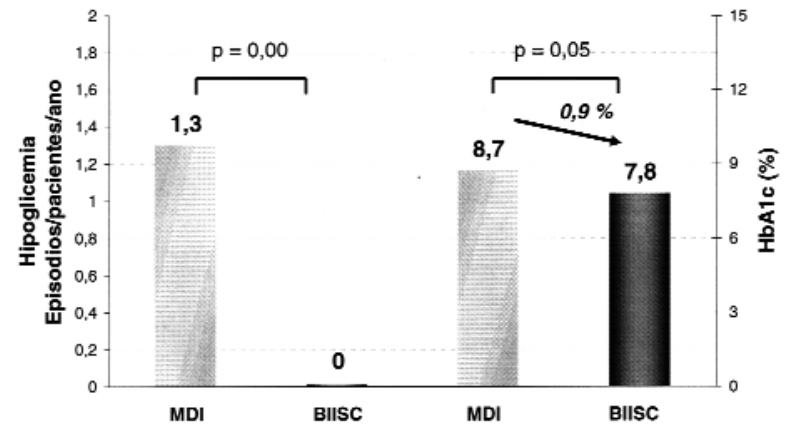

Figura 2. Comparação entre a média dos episódios de hipoglicemia grave e dos valores de $\mathrm{HbA} 1 \mathrm{c}$ durante os dois tipos de tratamento nos indivíduos com DM1 estudados.

glucagon ou glicose intravenosa. Outros autores acreditam que a BIISC só corrige um dos fatores responsáveis pela hipoglicemia, que é a concentração inadequada de insulina plasmática, não atuando nos demais elementos que contribuem para glicemia, o que restringe o seu uso universal (22).

Nossos resultados corroboram os encontrados na literatura, já que nenhum dos nossos pacientes apresentou hipoglicemia grave após mudança da terapia de MDI ( 1,5 episódio/ paciente/ ano) para BIISC (zero episódio/ paciente/ ano). Um terço dos pacientes apresentaram episódios isolados de hipoglicemia leve $(50-70 \mathrm{mg} / \mathrm{dl})$, geralmente após as refeições, devido a erros na contagem de carboidrato ou por hipercorreção das glicemias pré-refeição.

Em relação a controle metabólico, nossos pacientes atingiram a média de $\mathrm{HbAlc}$ de $7,9 \%$ que é similar à média descrita para os adolescentes do DCCT $(8,1 \%)$, com uma queda de $0,9 \%$ comparada ao período com MDI. A queda foi mais significativa nos pacientes com pior controle prévio e foi melhor do que a obtida em uma metanálise de bomba de insulina $(0,5 \%)(23)$ ou de um estudo em crianças que não encontrou nenhuma diferença no controle metabólico entre as terapias de MDI ou BIISC (24). Outro estudo também encontrou uma queda de $0,9 \%$ na $\mathrm{HbAlc}$ em 24 adolescentes após 1 ano de tratamento com bomba, assim como diminuição na dose de insulina e no número de hipoglicemia grave, no entanto seus pacientes apresentaram um aumento do IMC. Em dois dos nossos pacientes, a queda da $\mathrm{HbAl}$ c foi maior nos primeiros três meses de BIISC subindo depois para estabilizar-se num patamar maior, porém inferior aos valores iniciais. Este achado também foi relatado por
Plotinick e cols., que demonstraram o retorno dos valores da $\mathrm{HbAl}$ c inicial após a queda no primeiro ano de tratamento, após o terceiro ano de terapia com BIISC (25). Por outro lado, um estudo israelense com 279 pacientes estratificados por faixa etária (pré-púbere, púbere e adulto jovem) demonstrou que a queda da HbAlc foi maior nos adultos seguidos dos prépúberes e finalmente os adolescentes, e que houve um incremento adicional na queda da HbAlc para cada ano a mais de terapia de BIISC até terceiro ano de uso (26). Em um estudo no Barbara Davis Center for Childhood Diabetes, 56 adolescentes foram estratificados de acordo com a mudança $\geq 0,5 \%$ na HbAlc (decrescente, estável e crescente). Dezenove porcento desses jovens apresentaram aumento da HbAlc que foi justificado pela omissão freqüente dos bolus, mas 81\% apresentaram queda ou manutenção da HbAlc (27). Nossos dados não nos permitem afirmar que a BIISC produza um controle metabólico melhor que a terapia com MDI, devido às limitações estatísticas impostas pelo reduzido número de pacientes analisados e por servirem os mesmos como grupo controle.

Um dado relevante foi a redução na dose diária de insulina $(0,26 \mathrm{U} / \mathrm{kg} / \mathrm{dia})$, que contribuiu para que os pacientes não apresentassem ganho excessivo de peso, efeito adverso comum da terapia intensiva. Uma possível explicação é que a diminuição dos episódios de hipoglicemia diminuiu a ingestão de calorias extra para corrigir a queda da glicemia. Quanto à dose basal de insulina, esta variou de 40 a $60 \%$ da dose total, sendo que a taxa máxima liberada ocorreu em $57 \%$ dos pacientes no período noturno (17-23 horas), semelhante a outros estudos e diferente dos dados em adultos, cujo horário de taxa máxima ocorre entre $4-8$ horas da manhã (28).

Como é de conhecimento geral em relação à contagem de $\mathrm{CH}$, esta variou muito entre os pacientes (1U cada 6-20 gramas de $\mathrm{CH}$ ) e no mesmo paciente (maior necessidade no café que no jantar). Também houve variação entre os pacientes quanto ao fator de sensibilidade para correção de hiperglicemia, variando de $1 \mathrm{U}$ a cada $30-50 \mathrm{mg} / \mathrm{dl}$, além do alvo (glicemia de 80 a $120 \mathrm{mg} / \mathrm{dl}$ no jejum e $80-140 \mathrm{mg} / \mathrm{dl}$ pósprandial).

Estudos avaliando custo-benefício da BIISC concluem que os pacientes que mais se beneficiam da sua utilização são aqueles que apresentavam hipoglicemias severas $(29,30)$.

Em um editorial recente, John Pickup revela que 5\% dos pacientes DMl em terapia MDI apresentam hipoglicemia severa recorrente e outros $5 \%$ apresentam hipoglicemia severa que prejudicam a atividade 
diária (31); como somente $5 \%$ do total de pacientes DMl apresentam 70\% dos episódios graves de hipoglicemia, estes seriam os maiores beneficiários do uso de BIISC (1).

O relato desses casos apresenta a possibilidade da utilização da BIISC para prevenção de episódios de hipoglicemia grave em pacientes com DMl nas condições disponíveis em um serviço público de saúde em nível terciário. Paralelamente, obteve-se uma melhora significativa nos valores de HbAlc e redução nas necessidades diárias de insulina, fatores com reconhecida implicação na qualidade de vida e prognóstico a longo prazo desses adolescentes.

Entretanto, os resultados positivos obtidos em nosso centro precisam ser reproduzidos em centros semelhantes e estudos de custo são necessários para que a sua viabilidade seja confirmada e aplicada quando necessária para os pacientes com DMl assistidos nos sistemas públicos de saúde, que correspondem à maioria no nosso país.

\section{AGRADECIMENTOS}

À Medtronic do Brasil pelo fornecimento das BIISC, Minimed 508C, no período inicial do tratamento.

\section{REFERÊNCIA}

1. Pedersen-Bjergarard U, Pramming S, Heller SR, Wallace TM, Rasmusen AK, Jorgensen HV, et al. Severe hypoglycaemia in 1,076 adult patients with type 1 diabetes: influence of risk markers and selection. Diabetes Metab Res Rev 2004; $20: 476-86$.

2. Nordfeldt $S$, Jonsson D. Short-term effects of severe hypoglycaemia in children and adolescents with type 1 diabetes. A cost-of-illness study. Acta Paediat 2001;90:137-42.

3. Bulsara MK, Holman CDJ, Davis EA, Jones TW. The impact of a decade of changing treatment on rates of severe hypoglycemia in a population-based cohort of children with type 1 diabetes. Diabetes Care 2004;27:2293-8.

4. The Diabetes Control and Complications Trial Research Group. Hypoglycemia in the Diabetes Control and Complications Trial. Diabetes 1997;46:271-86.

5. Frey MA, Ellis D, Naar-King S, Greger N. Diabetes management in adolescents in poor metabolic control. Diabetes Educ 2004:30:647-57.

6. Boland A, Ahern D, Vincent R, Tamborlane W. Insulin pump therapy in pediatrics: A therapeutic alternative to safely lower HbA1c levels across all age groups. Pediatr Diabetes 2002;3:10-5.

7. Litton J, Rice A, Friedman N, Oden J, Lee M, Freemerk M. Insulin pump therapy in toddlers and preschool children with type 1 diabetes mellitus. J Pediatr 2002;141:490-5.

8. McMahon S, Airey L, Marangou D, McElwee K, Carne C, Clarey A, et al. Insulin pump therapy in children and adolescents: improvements in key parameters of diabetes management including quality of life. Diabet Med 2005;22:92-6.

9. Cogen FR, Streisand R, Sarin S. Selecting children and adolescents for Insulin Pump Therapy: Medical and behavioral considerations. Diab Spect 2002; 15:72-5.
10. Warren E, Weatherley-Jones E, Chilcott J, Beverley C. Systematic review and economic evaluation of a long-acting insulin analogue, insulin glargine. Health Technol Assess 2004;8:1-57.

11. Raskin P, Klaff L, Bergenstal R, Halle J-P, Donley D, Mecca T: A 16-week comparison of the novel insulin analog insulin glargine (HOE 901) and NPH human insulin used with insulin lispro in patients with type 1 diabetes. Diabetes Care 2000;23:1666-71.

12. Hermansen K, Fontaine P, Kukolja KK, Peterkova V, Leth G, Gall MA. Insulin analogues (insulin detemir and insulin aspart) versus traditional human insulins (NPH and regular human insulin) in basal-bolus therapy for patients with type 1 diabetes. Diabetologia 2004;47:622-9.

13. Dolyle EA, Wensimer SA, Steffen AT, Ahern JA, Vincent N, Tamborlane WV. A randomised prospective trial comparing the efficacy of continuous subcutaneous insulin infusion with multiple daily injections using insulin Glargine. Diabetes Care 2004:27:1554-8.

14. Hirsch IB, Bode BW, Garg S, Lane WS, Sussman A, HU P, et al. Continuous subcutaneus insulin infusion (CSII) of insulin aspart versus multiple daily injections of insulin aspart/glargine in type 1 diabetic patients previously untreated with CSII. Diabetes Care 2005;28:533-8.

15. Bolli GB, Capani F, Home PD, Kerr D, Thomas R, Torlone E, et al. Comparison of a multiple daily injection regimen with once daily insulin glargine basal insulin and mealtime lispro, continuous subcutaneous insulin infusion: a radomized, open, parallel study. Diabetes 2004;53(suppl 2):A107.

16. Liberatore Rdel R Jr, Damiani D. Insulin pump therapy in type 1 diabetes mellitus. J Pediatr (Rio J) 2006;82:249-54.

17. Malerbi D, Damiani D, Rassi N, Chacra AR, Niclewiccz EA, Silva Filho RL, et al. Posição de Consenso da Sociedade Brasileira de Diabetes - Insulinoterapia intensiva e terapêutica com bombas de insulina. Arq Bras Endocrinol Metab 2006;50/1:125-35.

18. Bode BW, Steed RD, Davidson PC. Reduction in severe hypoglycemia in long-term continuous subcutaneuos infusion in type 1 diabetes. Diabetes Care 1996;19:324-36.

19. Boland EA, Grey M, Oesterle A, Fredrickson L, Tamborlane WV. Continuous subcutaneuos insulin infusion: a new way to lower risk of severe hypoglycemia, improve metabolic control and enhance coping in adolescents with type 1 diabetes. Diabetes Care 1999;22:1779-84.

20. Linkeschova R, Raoul M, Bott U, Berg M, Spraul M. Less severe hypoglycemia, better metabolic control and improved quality of life in type 1 diabetes mellitus with continuous subcutaneuos insulin infusion (CSII) therapy: an observational study of 100 consecutive patients followed for a mean of 2 years. Diabet Med 2002;19:746-51.

21. Pickup J, Mattock M, Kerry S. Glycaemic control with continuous subcutaneous insulin infusion compared with intensive insulin injections in patients with type 1 diabetes: meta-analysis of randomised controlled trials. BMJ 2002;324:1-6.

22. Schade G, Valentine V. Are insulin pumps underutilized in type 1 diabetes? No. Diabetes Care 2006;29:1453-5.

23. Weissberg -Benchell J, Antisdel-Lomaglio J, Seshadri R. Insulin pump therapy - A meta-analysis. Diabetes Care 2003;26:1079-87.

24. Weintrob N, Benzaquen H, Galatzer A, Shalitin S, Lazar L, Fayman G, et al. Comparison of continuous subcutaneous insulin infusion and multiple daily injection regimens in children with type 1 diabetes: a randomized open crossover trial. Pediatrics 2003;112:559-64.

25. Plotnick LP, Clark LM, Brancati FL, Erlinger T. Safety and effectiveness of insulin pump therapy in children and adolescents with type 1 diabetes. Diabetes Care 2003:26:1142-6.

26. Nimri R, Weintrob N, Benzaquen H, Ofan R, Fayman G, Phillip $M$. Insulin pump therapy in youth with type 1 diabetes: a retrospective paired study. Pediatrics 2006;117:2126-31.

27. Maniatis AK, Klingensmith GJ, Slover R, Mowry CJ, Chase P. Continuous subcutaneous insulin infusion therapy for children and adolescents: An option for routine diabetes care. Pediatrics 2001;107:351-6. 
28. Conrad S, McGrath MT, Gitelman S. Transition from multiple daily injections to continuous subcutaneous insulin in type 1 diabetes mellitus. J Pediatr 2002;140:235-40.

29. Scuffham $P$, Carr L. The cost-effectiveness of continuous subcutaneous insulin infusion compared with multiple daily injections for the management of diabetes. Diabetes Med 2003;20:586-93.

30. Pickup J. Are insulin pumps underutilized in type 1 diabetes? Yes. Diabetes Care 2006;29:1449-52.

31. Feltbower RG, Campbell FM, Bodansky HJ, Stephenson CR, McKinney PA. Insulin pump therapy in childhood diabetes Cost implications for primary care trusts. Diabetes Med 2006;23(1):86-9.
Endereço para correspondência:

Mônica Andrade Lima Gabbay

Rua Baluarte 583, 71

04549-012 São Paulo, SP

Fax: (1 1 ) 5579-3590

E-mail: gabbay@ajato.com.br 